Vietnam Journal of Mechanics, VAST, Vol. 33, No. 4 (2011), pp. $283-291$

\title{
ABOUT THE CHOICE OF A PLASTIC-LIKE MODEL FOR SHAPE MEMORY ALLOYS
}

\author{
C. Lexcellent, R. M. Laydi \\ Département DMA, FEMTO-ST (UMR CNRS 6174) \\ University of Franche-Comté, France
}

\begin{abstract}
The aim of this paper is to examine the impact of the choice of plasticity theory-inspired model in the prediction of the shape of phase transformation domains. In this field a comparison is made between Huber-Von Mises based model and an another integrating the non-symmetry between tension and compression. The yield surface of phase transformation initiation for an homogeneous body under proportional biaxial loading is discussed. A transport of these surfaces in the space of the "effective transformation strain of martensite tensor" is given.
\end{abstract}

Key words: Shape memory alloys, phase change, elastic-plastic models, multiaxial behavior.

\section{INTRODUCTION}

The thermomechanical behavior of shape memory alloys SMA can be considered as surprizing towards elasto-plastic classical materials. Two mechanisms can be involved in its loading response i.e. (i) a phase transformation between a mother phase called austenite (generally cubic) and a product phase called martensite (orthorhombic, monoclinic, tetragonal... ) (ii) a reorientation process of the martensite platelets. The phase transformation induced by stress action is called "pseudo-elasticity" and the reorientation from self accommodating martensite variants to stress induced ones is said "pseudo-plasticity". Apparently on loading, for instance, the tensile curve $\sigma \leftrightarrow \varepsilon$ are the sames than the ones for elastoplastic materials but not the mechanisms involved.

The differentiation comes on unloading. For SMA pseudo-elasticity, the behavior is reversible mechanically and irreversible for elasto-plastic (presence of residual plastic strain at stress free state).

The comparison on unloading between pseudo-plastic and elasto-plastic material is the presence of caoutchoutic effect for SMA and linear decrease for the classical material.

Concerning the SMA thermomechanical modeling in the litterature, it is out of question to cite all the works concerning the subject (around 2000 references). But there is three different scales of investigation (microscopic, mesoscopic and macroscopic). At microscale, with the crystallographical theory of martensite called CTM [2, 3], a special attention is devoted to the nature of the microstructure i.e. an exact interface between 
austenite and a single variant of martensite or a twinned martensite in front of austenite. The micro-macro models [12] combine micromechanical data (martensite variants, habit plane... and thermodynamical tools). Hence, starting from local constitutive equations, an efficient homogeneization technique permits to obtain the macro-quantities with the classical concept of Representative Volume Element RVE. In the macro-models, all the quantities are defined at the macroscale i.e. the stress tensor, the strain tensors (total one, phase transformation, elastic...). These ones are often called "models with internal variables". The physical choice of the internal variables (for example the volume fraction of martensite...) permits to make the distinction between those models and also their kinetic equations.

Without entering too much in the details a recent review concerning the modeling of SMA polycrystals can be found in [7].

Here, we focussed our interest on some "plasticity theory-inspired SMA models", cf. for example [1, 19] and specially on [15] and also [6] investigations.

As in the plasticity theory, an important tool is the determination of the yield surfaces.

Some Huber-Von Mises models called $J_{2}$ ones can be chosen where the obvious non-symmetry between tension and compression is neglected (see for instance [11]).

But as the non-symmetry between tension and compression is experimentaly observed and measured ([18] on copper based alloys and [10] on $\mathrm{Ni} \mathrm{Ti}$ ), one has to take into account this important data in the modeling. In this aim, [15] have built a tridimensional model integrating this experimental information.

For example, the phase transformation yield surfaces (or reorientation ones) are no more, for a biaxial loading, circles in the stress deviatoric but new convex surfaces.

Bicompression and tension(compression)-internal pressure tests on a CuAlBe polycrystal permits to [5] to determine the shape of these new surfaces.

But a question arises, what is the impact of the 3D model choice (integrating or not the non-symmetry between tension and compression) on the phase transformation yield surfaces shape prediction for a proportional loading on an homogeneous SMAs sample?

In the present paper, one would make a comparison of the predictions delivered by the two types of 3D thermomechanical model.

\section{DETERMINATION OF THE YIELD SURFACE OF PHASE TRANSFORMATION FOR AN HOMOGENEOUS SMA BODY}

At first, the SMA are considered as pressure-insensitive alloys. It means that the phase transformation between austenite and martensite is accompanied with no volume change. As pointed out by [15], the dependence of the yield criterion on the first invariant of the stress tensor $\underline{\sigma}\left(I_{1} \equiv \operatorname{tr} \underline{\sigma}\right)$ is negligible.

Only the second and third invariants of the stress deviator are involved. As for the plasticity initiation, in the tensor $\underline{\sigma}$, the material is only sensitive to the stress deviator $\underline{S}_{\sigma} \equiv \operatorname{dev} \underline{\sigma}$.

The present analysis is restricted to materials with isotropic elastic behavior. 
Let us consider the second order symmetric stress tensor $\underline{\sigma}$ and the deviatoric tensor $\underline{\mathrm{S}}_{\sigma}$ defined by :

$$
\underline{S}_{\sigma} \equiv \underline{\sigma}-\frac{1}{3} \operatorname{tr} \underline{\sigma 1}
$$

In a classical way, the Huber-Von Mises equivalent stress $\bar{\sigma}$ is defined by :

$$
\bar{\sigma}=\kappa\left|\underline{S}_{\sigma}\right|, \quad \kappa=\sqrt{\frac{3}{2}}, \quad\left|\underline{S}_{\sigma}\right|=\left|\underline{S}_{\sigma}: \underline{S}_{\sigma}\right|^{1 / 2}
$$

The yield surface equation of phase transformation initiation (Austenite $\mathrm{A} \rightarrow$ Martensite $\mathrm{M}$ ) reads

$$
F(\underline{\sigma})=\bar{\sigma} f\left(x_{\sigma}\right)=b\left(T-M_{s}\right)=\sigma_{c}
$$

where $f>0$, is a smooth function (its second derivative is continuous) defined in the interval $I_{x}$ (to be defined later). In fact, $\mathrm{f}$ is introduced to take into account the nonsymmetry between tension and compression called "stress differential effect" by [16]. In $[13,14]$ is referred as "the shape function".

$\sigma_{c}$ is considered as the yield stress for phase transformation initiation.

$M_{s}$ : the martensitic start temperature with stress free state.

$b:$ the slope of the yield stress dependance with temperature.

$x_{\sigma}$ can have three different definitions

$$
x_{\sigma}=\left\{\begin{array}{l}
\text { The Lode invariant } y_{\sigma}: \quad y_{\sigma} \equiv 4 \operatorname{det}\left(N_{\sigma}\right) \in J_{y \sigma}[-1,1]\left(\underline{N}_{\sigma}=\frac{\kappa^{2} S_{\sigma}}{\bar{\sigma}}\right) \\
\text { The Lode angle } \theta_{\theta}: \quad \theta_{\theta}=\frac{1}{3} \arccos y_{\sigma} \in J_{\theta_{\sigma}}=\left[0, \frac{\pi}{3}\right] \\
\rho_{\sigma} \equiv \cos \left(\frac{1}{3} \arccos y_{\sigma}\right) \in I_{\rho_{\sigma}} \equiv\left[\frac{1}{2}, 1\right]
\end{array}\right.
$$

where $\rho_{\sigma}$ constitutes the greatest eigenvalue of $\underline{\mathrm{N}}_{\sigma}$.

If one introduces the $\mathrm{g}$ function with the following definition

$$
g\left(\rho_{\sigma}\right)=-\rho_{\sigma} \frac{d f}{d \rho_{\sigma}}+f\left(\rho_{\sigma}\right)
$$

[9] demonstrates the convexity conditions of the yield function $F$ as

$$
\left\{\begin{array}{c}
g\left(\rho_{\sigma}\right) \geq 0 \\
\frac{d g}{d \rho_{\sigma}} \leq 0
\end{array}\right.
$$

for all $\rho_{\sigma} \in I_{\rho_{\sigma}}$.

With some calculations, one can find the conditions given in terms of the Lode invariant $y_{\sigma}$ by $[16]$

$$
\frac{f\left(y_{\sigma}\right)}{9}-y_{\sigma} f^{\prime}\left(y_{\sigma}\right)+\left(1-y_{\sigma}^{2}\right) f^{\prime \prime}\left(y_{\sigma}\right) \geq 0, \quad \forall y_{\sigma} \in[-1,1]
$$

And also the conditions in terms of the Lode angle $\theta_{\sigma}$ given by [4]

$$
f_{*}^{2}\left(\theta_{\sigma}\right)+2 f_{*}^{\prime}\left(\theta_{\sigma}\right)-f_{*}^{\prime \prime}\left(\theta_{\sigma}\right) f\left(\theta_{\sigma}\right) \geq 0, \quad \forall \theta_{\sigma} \in\left[0, \frac{\pi}{3}\right]
$$

with $f_{*}=f^{-1}\left(\theta_{\sigma}\right)$. 
By duality and transport, one can built the yield surface in the space of "the effective transformation strain of martensite" $\underline{\varepsilon}_{m}$ [17]. This concept of $\underline{\varepsilon}_{m}$ constitutes the average transformation strain of the different variants averaged over a representative volume containing multiple grains, after material has formed on allowable microstructure.

By definition, the total strain tensor $\underline{\varepsilon}$ can be written as :

$$
\underline{\varepsilon}=\underline{\varepsilon}_{e}+\underline{\varepsilon}_{m} z, \quad z \in[0,1],
$$

where $z$ constitutes the volume fraction of martensite inside the mother phase (austenite) and $\underline{\varepsilon}_{e}$ the elastic deformation.

For proportional loadings, the experiments show that the phase transformation strain rate is normal to the yield surface $F(\underline{\sigma})[5]$ (Fig. 1).

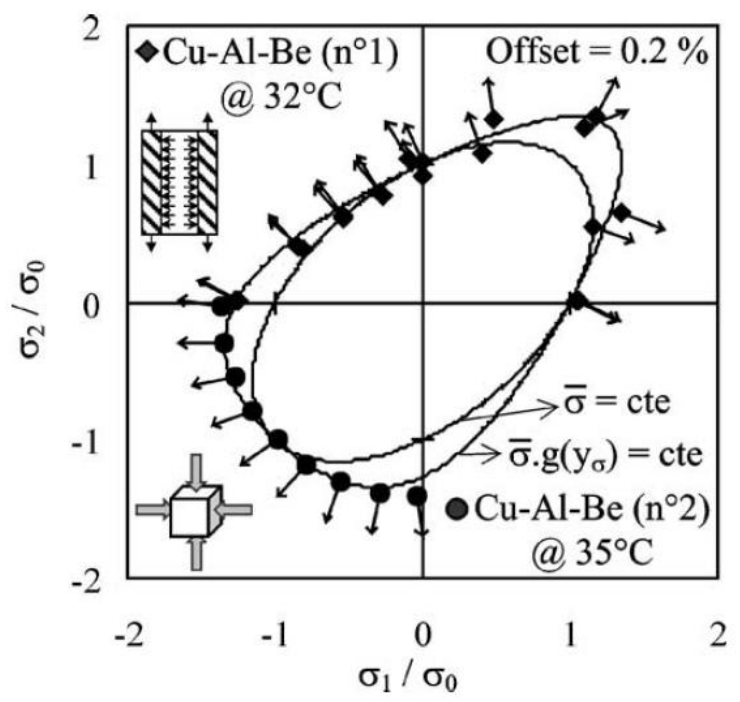

Fig. 1. Experimental phase transformation onset stress under biaxial proportional loading and modeling. Experimental proof of the normality rule $\left(\mathrm{Cu} \mathrm{Al} \mathrm{Be}\left(\mathrm{n}^{\circ} 1\right)\right.$ : $\checkmark$ tube for tension-compression internal pressure $\mathrm{Cu} \mathrm{Al} \mathrm{Be}\left(n^{\circ} 2\right)$, • cube for bicompression

Thus, one can assume that $\underline{\varepsilon}_{m}(\underline{\sigma})$ admits a potential $(F \underline{\sigma})>0$ which means

$$
\underline{\varepsilon}_{m}=\gamma F^{\prime}(\underline{\sigma})
$$

The calculations of [9] show that

$$
\frac{\underline{\varepsilon}_{m}}{\gamma}=f\left(x_{\sigma}\right) \underline{N}_{\sigma}+a(x) f^{\prime}\left(x_{\sigma}\right) \underline{N}_{\sigma}^{\perp}
$$

where

$$
\underline{N}_{\sigma}^{\perp}=\left(1-\rho_{\sigma}^{2}\right) \underline{e}_{1}^{R \sigma}+\rho_{\sigma} \underline{e}_{2}^{R \sigma}
$$

and $\gamma$ is the maximum shear phase transformation strain.

$e_{1}^{R \sigma}$ and $e_{2}^{R \sigma}$ are two elementary deviatoric associated tensors to $\underline{\sigma}$ by

$$
\underline{e}_{1}^{R \sigma}=\underline{R}_{\sigma} \underline{e}_{1} \underline{R}_{\sigma}^{T} \text { and } \underline{e}_{2}^{R \sigma}=\underline{R}_{\sigma} \underline{e}_{2} \underline{R}_{\sigma}^{T}
$$


where $\underline{R}_{\sigma}$ is the rotation matrix transforming $\underline{\sigma}$ in its diagonal form.

$$
\begin{aligned}
& \underline{e}_{1}=\left(\begin{array}{ccc}
\frac{-1}{2} & 0 & 0 \\
0 & \frac{-1}{2} & 0 \\
0 & 0 & 0
\end{array}\right) ; \quad \underline{e}_{2}=\left(\begin{array}{lll}
\frac{\sqrt{3}}{2} & 0 & 0 \\
0 & \frac{-\sqrt{3}}{2} & 0 \\
0 & 0 & 0
\end{array}\right) ; \\
& a(x)=\frac{d x}{d \rho}\left(1-|\rho(x)|^{2}\right)^{1 / 2}= \begin{cases}3\left(1-x^{2}\right)^{1 / 2} & \text { if } x=y_{\sigma} \\
-1 & \text { if } x=\theta_{\sigma} \\
\left(1-x^{2}\right)^{1 / 2} & \text { if } x=\rho_{\sigma}\end{cases}
\end{aligned}
$$

and

$$
\frac{\varepsilon_{m}}{\gamma}= \begin{cases}f\left(y_{\sigma}\right) \underline{N}_{\sigma}+3 f^{\prime}\left(y_{\sigma}\right)\left(2 \underline{N}_{\sigma}^{2}-y_{\sigma} \underline{N}_{\sigma}-\underline{1}\right) & \text { for } x=y_{\sigma} \\ f\left(\theta_{\sigma}\right) \underline{N}_{\sigma}-f^{\prime}\left(\theta_{\sigma}\right) \underline{N}_{\sigma}^{\perp} & \text { for } x=\theta_{\sigma} \\ f\left(\rho_{\sigma}\right) \underline{N}_{\sigma}+\left(1-\rho_{\sigma}\right)^{1 / 2} f^{\prime}\left(\rho_{\sigma}\right) \underline{N}_{\sigma}^{\perp} & \text { for } x=\rho_{\sigma}\end{cases}
$$

Under the hypothesis providing the univocal correspondance between $\underline{\sigma}$ and $\underline{\varepsilon}_{m}$, one can write the equation of the phase transformation surface as $([6])$

$$
H\left(\underline{\varepsilon}_{m}\right)=\bar{\varepsilon}_{m} h\left(x_{\varepsilon}\right)=\gamma
$$

where

$$
\bar{\varepsilon}_{m}=\kappa\left|\underline{\varepsilon}_{m}\right|
$$

and

with

$$
h\left(x_{\varepsilon}\right)=\frac{\kappa^{-2}}{\delta_{f}\left(x_{\sigma}\right)},
$$

$$
\delta_{f}\left(x_{\sigma}\right)=\left(\left|f\left(x_{\sigma}\right)\right|^{2}+\left|a\left(x_{\sigma}\right)\right|^{2}\left|f^{\prime}\left(x_{\sigma}\right)\right|^{2}\right)^{1 / 2} .
$$

As applications, we consider two experimental series concerning $\mathrm{Cu}-\mathrm{Al}-\mathrm{Be}$ and NiTi alloys, whose yield values are given (in Mpa) in Table 1 and Table 2.

Table 1

\begin{tabular}{|c|c|c|c|c|c|c|c|c|c|c|c|}
\hline$I$ & 1 & 2 & 3 & 4 & 5 & 6 & 7 & 8 & 9 & 10 & 11 \\
\hline$\sigma_{1}$ & 87.14 & 91.43 & 105.71 & 45.71 & 0 & -45.71 & -105.71 & -100 & -65.71 & 0 & 51.43 \\
\hline$\sigma_{2}$ & 0 & 42.86 & 102.86 & 100 & 80 & 60 & 0 & -48.57 & -91.43 & -100 & -48.57 \\
\hline
\end{tabular}

Table 2

\begin{tabular}{|c|c|c|c|c|c|c|c|c|c|c|c|c|c|c|c|}
\hline$I$ & 1 & 2 & 3 & 4 & 5 & 6 & 7 & 8 & 9 & 10 & 11 & 12 & 13 & 14 & 15 \\
\hline$\sigma_{z z}$ & 400 & 390 & 310 & 220 & 0 & -210 & -370 & -520 & -530 & -520 & -430 & -200 & 30 & 250 & 370 \\
\hline$\sigma_{z \theta}$ & 0 & 150 & 240 & 340 & 440 & 420 & 330 & 220 & 0 & -150 & -340 & -400 & -370 & -300 & -150 \\
\hline
\end{tabular}


Concerning the $\mathrm{Cu}-\mathrm{Al}-\mathrm{Be}$ alloy, the yield points are obtained by bi - compression tests on cubes and tension (compression) - internal pressure on tubes (see [5])

$$
\mathrm{Cu}-\mathrm{Al}-\mathrm{Be}: \underline{\sigma}_{i}=\left(\begin{array}{ccc}
\sigma_{1} & 0 & 0 \\
0 & \sigma_{2} & 0 \\
0 & 0 & 0
\end{array}\right), \quad 1 \leq i \leq n=11 .
$$

The stress numerical values are given in MPA.

As for the $\mathrm{Ni}$ - Ti alloy, the tension (compression) - torsion tests on tubes as performed by [8] give the following yield values :

$$
\text { NiTi }: \underline{\sigma}_{i}=\left(\begin{array}{ccc}
0 & 0 & 0 \\
0 & 0 & \sigma_{z \theta} \\
0 & \sigma_{z \theta} & \sigma_{z z}
\end{array}\right), \quad 1 \leq i \leq n=15 .
$$

We will use the shape function $\mathrm{f}$ which has been introduced in [5], namely

$$
f\left(y_{\sigma}\right)=\cos \left(\frac{1}{3} \arccos \left(1-a\left(1-y_{\sigma}\right)\right)\right) .
$$

One shows that $\mathrm{F}$ is convex $([9])$ if and only if

$$
0 \leq a \leq 1 .
$$

The Fig. 1 exhibits the yield surface in the stress space and the Fig. 2 its dual in the $\varepsilon_{m}$ space for CuAlBe alloy.

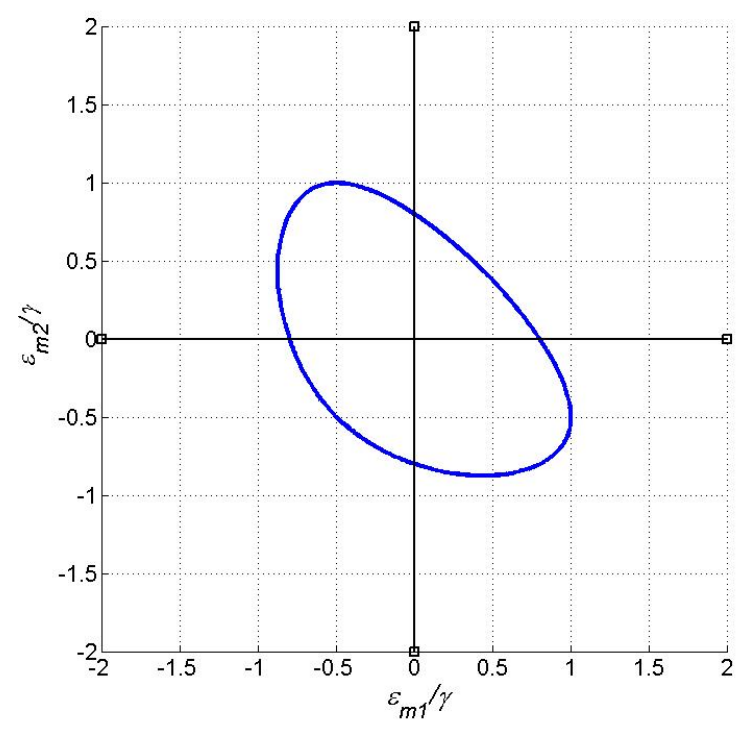

Fig. 2. Yield surface in the phase transformation strain dual space $\underline{\varepsilon}_{m}$

The Fig. 3 shows the yield surface in the stress space and the Fig. 4 its dual in the $\varepsilon_{m}$ space for $\mathrm{Ni}-\mathrm{Ti}$ alloys. 


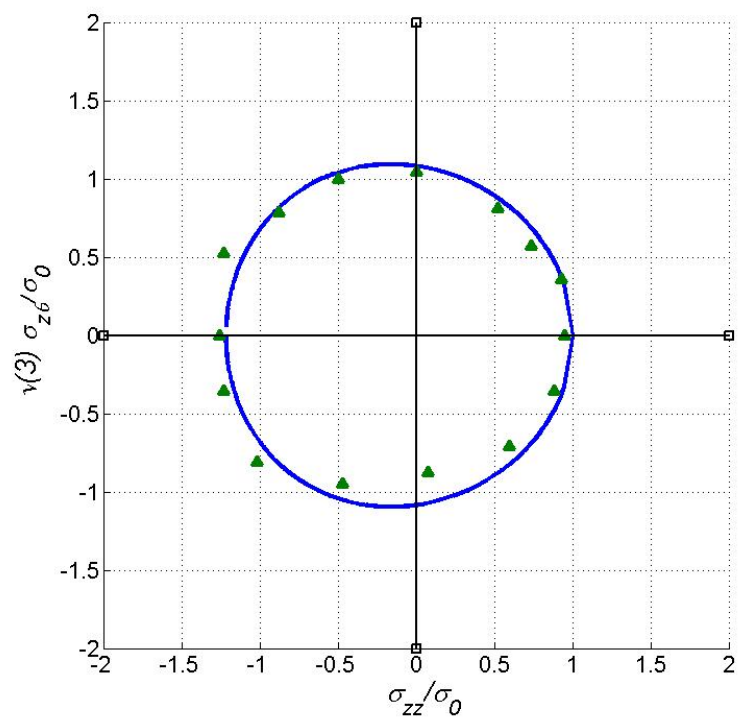

Fig. 3. Experimental phase transformation onset stress under tension(compression)-torsion biaxial proportional loading and modeling (NiTi, experimental points, - modeling)

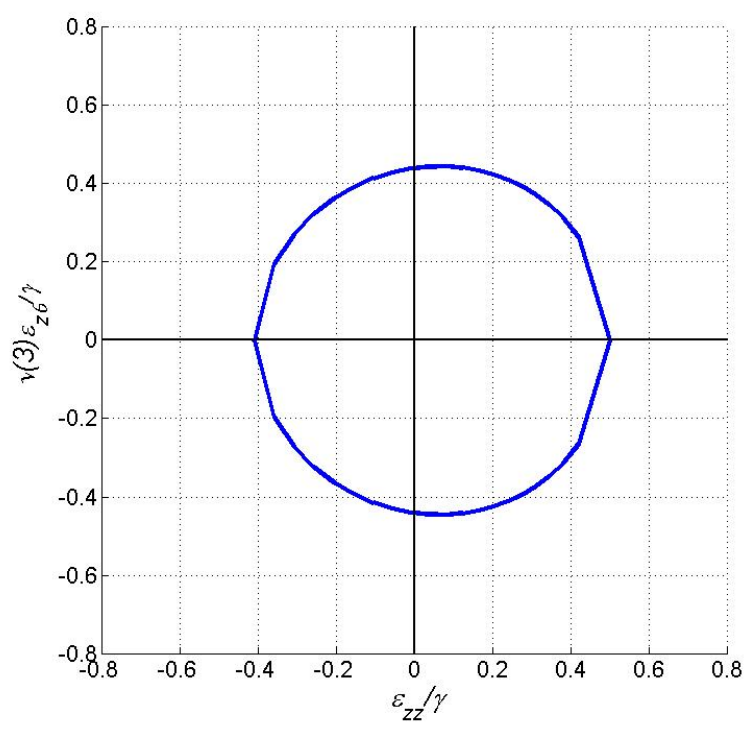

Fig. 4. Yield surface in the phase transformation strain dual space $\underline{\varepsilon}_{m}$ 
The yield stress measurements on the two alloys show that a model integrating the assymetry between tension and compression is totally necessary.

For copper based alloys and surely $\mathrm{NiTi}$, on loading the phase transformation is not complete and the detection of associated yield points not sure.

As the detection of the yield values for the reverse phase transformation $(M \rightarrow A)$ are also very delicate, the analysis on unloading is not made.

\section{CONCLUSION}

A thermomechanical modeling of shape memory alloys is performed here for tri dimensional loadings (cf. also [15]). This description includes the "stress differential effect" by integrating the assymetry between tension and compression in its prediction.

Some basic applications are discussed. For the description of the yield surface determination in the $2 \mathrm{D}$ situations, one moves classicaly from the circles for $\mathrm{J} 2$ model, to convex surfaces for (J2, J3) models. A surface transport can be made between the space of "effective transformation strain of martensite" $\varepsilon_{m}$ and the space of stress ([6]) and vice - versa.

\section{REFERENCES}

[1] Aurrichio F., Reali A., Stefanelli U., A three - dimensional model describing stress - induced solid phase transformation with permanent elasticity, Int. J. of Plasticity, 24(4), (2007), 207 $-226$.

[2] Ball J. M., James R. D., Fine phase mixtures as minimizers of energy, Arch. Ration. Mech. Anal., 100(1), (1987), 13 - 52.

[3] Ball J. M., James R. D., Proposed experimental tests of a theory of fine microstructure and the two wells problem, Philos. Trans. R. Soc. London, Ser. A 338, (1992), 389 - 450.

[4] Bigoni D., Piccolroaz A., Yield criteria for quasibrittle and functional materials, Int. J. of Solids and Struct., 41, (2004), 2855 - 2878.

[5] Bouvet C., Calloch S., Lexcellent C., Mechanical behavior of a $\mathrm{Cu}$ - Al - Be shape memory alloy under multiaxial proportional and non - proportional loadings, Journal of Engineering Material and Technology, 124, (2002), 112 - 124.

[6] Gibeau E., Laydi R. M., Lexcellent C., Determination and transport of phase transformation yield surfaces for shape memory alloys, Z. Angew. Math. Mech., 90(7-8), (2010), 595 - 604.

[7] Lagoudas D. C., Entchev P. B., Popov P., Patoor E., Brinson L. C., Gao X., Shape memory alloys - Part II : Modeling of polycrystals, Mech. of Mat., 38 (5-6), (2006), 430 - 462.

[8] Laverhne - Taillard K., Calloch S., Arbab Chirani S. and Lexcellent C., Multiaxial shape memory effect and superelasticity, Strain, 45(1), (2008), 77 - 84.

[9] Laydi R. M., Lexcellent C., Yield criteria for shape memory materials: convexity and surface transport, Math. Mech. Solids, 15, (2010), $165-208$.

[10] Orgeas L., Favier D., Stress - induced martensitic transformation of a Ni - Ti alloy in isothermal shear, tension and compression, Acta Materialia, 46(15), (1998), 5579 - 5591.

[11] Panoskaltis V. P., Bahugana S., Soldatos D., On the thermomechanical modeling of shape memory alloys, Int. J. Non - Linear Mech., 39(5), (2004), 709 - 722.

[12] Patoor E., Lagoudas D. C., Entchev P. L., Brinson L. C. and Gao X., Shape memory alloys - Part I: General properties and modeling of single crystals, Mech. of Mat., 38(5-6), (2006), $391-429$. 
[13] Podgorski J., Limit state condition and the dissipation function for isotropic materials, Arch. Mech., 36, (1984), 323 - 342.

[14] Podgorski J., General failure criterion for isotropic media, J. Engng. Mech. ASCE, 111(2), (1985), 188 - 199.

[15] Raniecki B., Lexcellent C., Thermodynamics of isotropic pseudoelasticity in shape memory alloys, Eur. J. Mech. A Solids, 17, (1998), $185-205$.

[16] Raniecki B. and Mroz S., Yield or martensitic phase transformation conditions and dissipation functions for isotropic, pressure - insensitive alloys exhibiting SD effect, Acta Mech., 195(1-4), (2008), 81 - 102.

[17] Sadjadpour A., Bhattacharya K., A micromechanics - inspired constitutive model for shape memory alloys, Smart Mater. Struct., 16, (2007), 1751 - 1765.

[18] Vacher P., Lexcellent C., Study of pseudoelastic behavior of polycrystalline SMA by resistivity measurements and acoustic emission, In Proc. Of ICM 6, Pergamon Press Editors Jono M. and Inoue T., (1991), 231 - 236.

[19] Zaki N., Moumni Z., A three - dimensional model of the thermomechanical behavior of shape memory alloys, J. Mech. Phys. Solids, 55, (2007), $2455-2490$. 